\title{
¿Son los Signos Duros de Lesión en Cuello Mandatorios de Exploración Quirúgica?
}

\author{
Martha Quiodettis, Otilda M Valderrama V, Aldo A Ávila, Ayusel Valle, Loyda Rodríguez
}

\begin{abstract}
RESUMEN
Introducción: Las lesiones en cuello siguen siendo un reto diagnóstico para los médicos de trauma. En la literatura se encuentran diversos algoritmos de manejo. En los últimos años, el abordaje de estos pacientes ha evolucionado con la utilización de diversas técnicas diagnósticas e incluso el manejo conservador de algunas lesiones venosas.
\end{abstract}

Objetivo: Determinar la relación entre los hallazgos clínicos y paraclínicos con las lesiones comprobadas mediante cirugía o estudios diagnósticos.

Métodos: Estudio Descriptivo Retrospectivo, se revisaron los expedientes de todos los pacientes admitidos con trauma en cuello entre el 2010 y 2011.

Resultados: Se analizaron 84 pacientes, $94.1 \%$ de sexo masculino, edad promedio de 30.4 años, e ISS de 8.8 . EI $98.8 \%$ con lesiones penetrantes, principalmente en Zona II (62.7\%). El $25.7 \%$ fueron llevados a exploración quirúrgica inmediata y el resto se les realizó estudios diagnósticos en el cuarto de urgencias. La incidencia de lesiones fue del $21.4 \%$, siendo la mayoría lesiones venosa... Al evaluar los pacientes que presentaron lesión vs que no presentaban, no se encontraron diferencias estadísticamente significativas $(p>0.05)$ en cuanto a mecanismo de lesión, inestabilidad hemodinámica, sangrado a través de la herida, trayecto vascular, enfisema subcutáneo y hematoma (expansivo o no). Se encontró diferencias significativas $(p<0.05)$ en cuanto a dificultad respiratoria OR 8.24 IC95\% (1.08-20.7) y necesidad de intubación en el cuarto de urgencias OR 4.0 IC95\% (1.32-12.1). La mortalidad fue de $2.38 \%$.

Conclusión: No todos los síntomas y signos en los pacientes con lesiones de cuello son predictivas de lesiones significativas que requieran cirugía.

Palabras claves: Lesiones de cuello, Signos duros, Signos blandos.

\section{ABSTRACT \\ Introduction: Neck injuries are challenging manage for surgeons. The literature has multiple treatment algorithms. In recent years, management of this patients has evolve using different diagnostic tools including nonoperative treatment in some venous injuries.}

Objective: Determine the relationship between findings in physical exam with the injuries found during surgery or in diagnostic tests.

Methods: We retrospectively reviewed the medical records of patients admitted with neck trauma between 2010 and 2011 in our service.

Results: Eighty four patients were analyzed, 94.1\% male, average age 30.4 years, median ISS 8.8 . A total of $98.8 \%$ presented penetrating injuries, mainly in zone II (62.7\%). Only $25.7 \%$ underwent immediate surgical exploration and the others had diagnostics test in the emergency room. The incidence of injuries was $21.4 \%$, vein injuries were more frequent. When comparing patients with injuries vs the ones with no lesions there were no difference in injury mechanism, vital signs on admission, bleeding trough the wound, vascular trajectory, subcutaneous emphysema or hematoma. There was a statiscally difference $(p<0.05)$ between respiratory distress (OR 8.24, IC 95\%, 1.08-20.7) and need for orotracheal intubation in ER (OR 4.0, IC 95\%, 1.32-12.1). Overall mortality was $2.38 \%$.

Conclusion: Not all the symptoms and signs in patients with traumatic injuries to the neck are predictive for surgical intervention.

Keywords: Neck injuries, Soft signs, Hard signs.

How to cite this article: Quiodettis M, Valderrama VOM, Ávila AA, Valle A, Rodríguez L. ¿Son los Signos Duros de Lesión en Cuello Mandatorios de Exploración Quirúgica? Panam J Trauma Critical Care Emerg Surg 2013;2(1):17-20.

\section{Source of support: Nil}

Conflict of interest: None declared

\section{INTRODUCCIÓN}

El manejo del trauma de cuello se modificó a través de los años. Inicialmente se realizaba exploración mandataria para todos los pacientes con lesiones en Zona II. Sin embargo, a través de los años, diversos estudios han demostrado que no todos los pacientes con lesiones en cuello ameritan una exploración quiúrgica. ${ }^{1}$ Esto ha llevado a que se han identificado los síntomas y signos con mayor probabilidad de presencia de lesión (Tabla 1) para guiarnos en la toma de decisión quirúrgica. ${ }^{1,2}$ Así, en la actualidad se prefiere una conducta menos invasiva utilizando métodos diagnósticos, en aquellos pacientes hemodinámicamente normales.

En nuestra institución el manejo usual es la exploración quirúrgica ante la presencia de síntomas de lesión a estructuras o inestabilidad hemodinámica. Sin embargo, se ha observado una baja incidencia de lesiones. Es por esto, que decidimos analizar los pacientes operados en los últimos años con el objetivo de determinar la relación entre los hallazgos clínicos y para clínicos con las lesiones comprobadas mediante cirugía o estudios diagnósticos.

Se trata de un estudio descriptivo, retrospectivo en el cual se incluyeron todos los pacientes admitidos al Servicio de Trauma del Hospital Santo Tomás con diagnóstico de trauma en cuello durante los años 2010 y 2011.

\section{METODOLOGÍA}

Se analizaron todos los expedientes de los pacientes admitidos con diagnóstico de trauma en cuello durante los 
años 2010 y 2011. Los pacientes fueron divididos en dos grupos: Pacientes en los cuales se encontró alguna lesión de estructuras (Grupo I) y pacientes en los que no se evidenció lesión (Grupo II).

Para determinar la presencia de lesiones, nos basamos en la presencia de las mismas diagnosticadas por estudios de gabinete o exploración quirúrgica.

Se creó una base de datos en el programa Epi Info 7 y el mismo también se utilizó para los análisis estadísticos (significancia estadística: $\mathrm{p}<0.05, \chi^{2}>3.85$ ).

\begin{tabular}{|c|c|}
\hline Signos 'duros' & Signos 'blandos' \\
\hline Compromiso de la vía aérea & Disfagia \\
\hline Shock o sangrado activo & Disfonía \\
\hline Hematoma pulsátil & Hemoptisis \\
\hline Enfisema subcutáneo extenso & Ensanchamiento de mediastino \\
\hline
\end{tabular}

\section{RESULTADOS}

Durante los años 2010 y 2011 se atendieron un total de 91 pacientes con trauma en cuello de los cuales se excluyeron 7 por expediente incompleto.

El 94.1\% masculinos, con promedio de edad de $30.4 \pm$ 12.9 años. El $57.4 \%$ se trataron de heridas por arma blanca, el $41.8 \%$ heridas por proyectil de arma de fuego (PAF) y sólo un caso de trauma cerrado.

El $63.1 \%$ fueron heridas en Zona II, seguidas por un $19.1 \%$ de heridas en Zona III y $17.9 \%$ de heridas en Zona I. El 29.7\% de los pacientes fueron llevados a exploración quirúrgica inmediata y al resto se le realizaron estudios siendo la Angio-tomografía la más utilizada (64.3\%).

Se encontraron lesiones en 34 pacientes (incidencia de $21.4 \%)$, más de la mitad de las mismas (55.9\%) se trataban de lesiones venosas. En la Tabla 2 se pueden observar las características generales de los dos grupos estudiados y en la Gráfica 1 se observan los tipos de lesiones.

Al evaluar los signos vitales y datos a su ingreso (Tabla 3) no se evidenció diferencia en cuanto a los datos hemodinámicos y escala de Glasgow. Se evidenció una mayor incidencia de lesiones en aquellos pacientes que ameritaron intubación en el cuarto de urgencias.

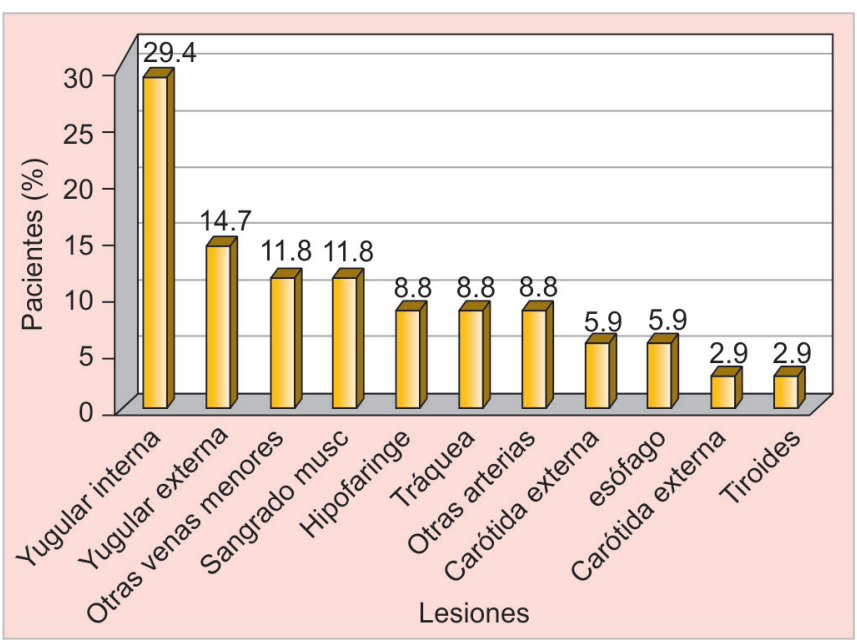

Gráfico 1: Lesiones encontradas en los pacientes con trauma en cuello

\begin{tabular}{|c|c|c|c|c|}
\hline & & Pacientes con lesión $(n=34)$ & Pacientes sin lesión $(n=50)$ & $p$-value \\
\hline \multicolumn{2}{|l|}{ Edad } & $33.5 \pm 15$ años $(16-79)$ & $28.6 \pm 11.5$ años $(15-75)$ & 0.16 \\
\hline \multicolumn{2}{|l|}{ ISS } & $11.5 \pm 11.1(1-49)$ & $7.05 \pm 7.3(1-35)$ & $\begin{array}{l}0.01 \\
\chi^{2}=6.89\end{array}$ \\
\hline \multirow[t]{4}{*}{ Mecanismo } & Arma blanca & $18(52.9 \%)$ & $30(60 \%)$ & 0.89 \\
\hline & $\mathrm{PAF}^{*}$ & $13(38.2 \%)$ & $22(44 \%)$ & 0.06 \\
\hline & Cerrado & $0(0 \%)$ & $1(2 \%)$ & 0.44 \\
\hline & 1 & $6(17.6 \%)$ & $9(18 \%)$ & 0.78 \\
\hline \multirow[t]{2}{*}{ Zona } & II & $19(55.9 \%)$ & $34(68 \%)$ & 0.79 \\
\hline & III & $6(17.6 \%)$ & $10(20 \%)$ & 0.96 \\
\hline
\end{tabular}

*Proyectil de arma de fuego

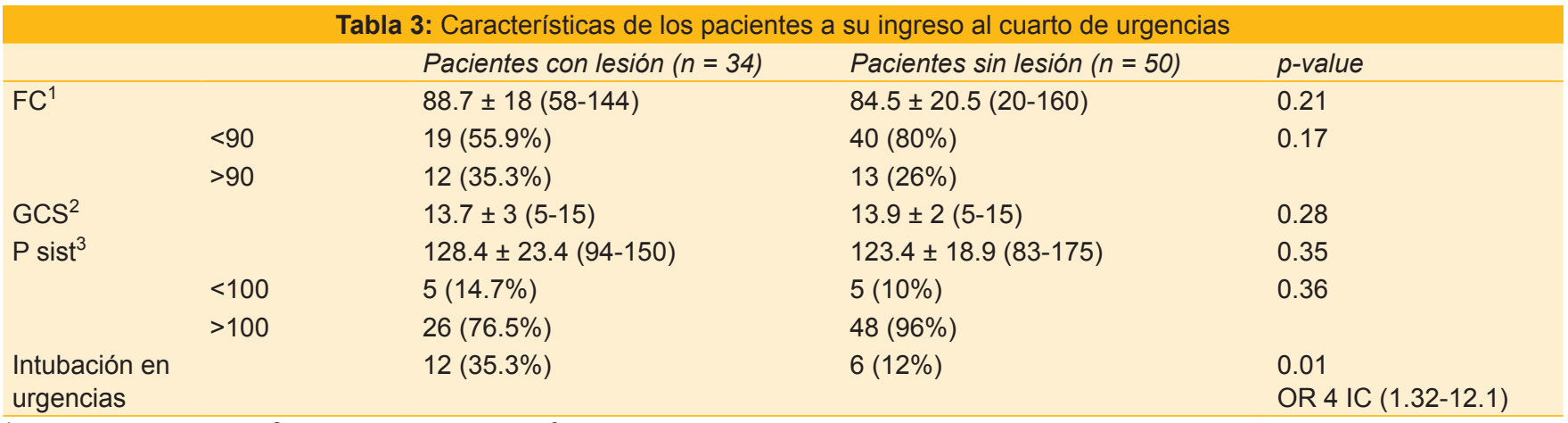

${ }^{1}$ Frecuencia Cardíaca; ${ }^{2}$ Glasgow Coma Scale; ${ }^{3}$ Presión sistólica 


\begin{tabular}{lccc} 
& Tabla 4: Características de los pacientes a su ingreso al cuarto de urgencias & \\
Hallazgos examen físico & $\begin{array}{c}\text { Pacientes con Lesión } \\
(n=34)\end{array}$ & $\begin{array}{c}\text { Pacientes sin Lesión } \\
(n=50)\end{array}$ & $p$-value \\
& $5(14.7 \%)$ & $1(2 \%)$ & 0.02 \\
Dificultad respiratoria & & & OR 8.24 (1.08-20.7) \\
Trayecto vascular & $3(8.8 \%)$ & $0(0 \%)$ & 0.3 \\
Sangrado activo & $10(29.4 \%)$ & $6(12 \%)$ & 0.047 \\
Enfisema subcutáneo & & $12(24 \%)$ & OR 3.06 (0.99-9.43) \\
Hematoma no expansivo & $8(23.5 \%)$ & $20(40 \%)$ & 0.84 \\
Hematoma expansivo & $16(47.1 \%)$ & $0(0 \%)$ & 0.34 \\
\hline
\end{tabular}

En cuanto a los signos duros y blandos de lesión (Tabla 4), sólo se observó una diferencia estadísticamente significativa de lesiones en aquellos pacientes con dificultad respiratoria, lo que se correlaciona con la necesidad de intubación.

La estancia hospitalaria promedio fue de 6.5 días (127) para los pacientes con lesión vs 5.3 días (1-58) para los pacientes sin lesión $(\mathrm{p}=0.46)$ debido a lesiones asociadas, principalmente intrabdominales. Sin embargo, tampoco se evidenció diferencia estadísticamente significativa al evaluar los pacientes sólo con trauma en cuello (3.3 vs 3.7 días; $\mathrm{p}=0.5$ ).

Se presentaron dos defunciones en el grupo de los pacientes con lesión $(5.9 \%)$ pero esta diferencia no fue estadísticamente significativa $(\mathrm{p}=0.83)$.

\section{DISCUSIÓN}

En 1995, Demetriades et al, ${ }^{4}$ describieron que aproximadamente el $35 \%$ de todas las lesiones por proyectil de arma de fuego y el $20 \%$ de las heridas por arma blanca están asociadas con lesiones significativas de órganos vitales, con sólo el 16.5 y $10.1 \%$ requiriendo una operación terapéutica. Hay otras series que reportan incidencias hasta un $89.7 \%$ de lesiones significativas. ${ }^{5,6}$

En nuestro estudio, encontramos una incidencia global de lesiones del 21.4\%. Más de la mitad de las mismas (55.9\%) se trataban de lesiones venosas, seguidas de sangrado muscular.

Clásicamente, se ha descrito que, los pacientes con signos ‘duros' de lesión deben ser llevados a exploración quirúrgica inmediata y que aquellos con signos 'blandos' se les debe realizar estudios diagnósticos. ${ }^{2,3}$

Esto se debe a que diversos estudios han descrito que la presencia de signos blandos de trauma vascular no identifica de manera efectiva a los pacientes que van a requerir una cirugía; sin embargo, la ausencia de los mismos tiene un valor predictivo negativo del $100 \%$ por lo que la excluye. ${ }^{1,4,7}$ En nuestra serie tampoco pudimos correlacionar estos hallazgos con la presencia de lesión (Tabla 3 y 4).
Probablemente esto se correlaciona con el hecho de la baja incidencia en nuestra serie de lesiones severas como lesión de arterias carótidas (9.8\%).

Sin embargo, en nuestro análisis de los signos 'duros', sólo pudimos observar una relación estadísticamente significativa [OR 8.24 (1.08-20.7)] para el compromiso de la vía aérea. Sabemos que esto debe ser analizado con cuidado ya que pacientes inestables hemodinámicamente (aunque no se correlacione en nuestra serie) no pueden ser llevados a estudios de extensión por lo que se debe continuar con su exploración quirúrgica.

Como hemos señalado previamente, la mayor incidencia de lesiones encontradas se trataba de las venas yugulares. En los últimos años se han publicado diversos estudios que advocan el manejo conservador o mínimamente invasivo de las mismas. ${ }^{6,8}$ Cabe entonces considerar si la presencia de hematomas en pacientes estables pueden ser manejados de manera más conservadora realizándoles angiotomografías a estos pacientes, ya que la probabilidad de lesión que requiera un manejo quirúrgico es baja.

$\mathrm{Si}$ a esto añadimos que un grupo de pacientes con lesiones de carótida se benefician de manejo endovascular (zonas de difícil acceso como la Zona I y, particularmente, Zona III) ${ }^{3,8}$ disminuimos aún más el número de pacientes que requerirán una cirugía por una lesión en cuello.

\section{CONCLUSIÓN}

Un examen físico adecuado con personas con experiencia en trauma de cuello y exámenes complementarios son necesarios para tomar decisiones terapéuticas. Se debe tener una alta sospecha de lesiones de estructuras del cuello en aquellos pacientes con dificultad respiratoria y necesidad de intubación a su ingreso.

\section{REFERENCIAS}

1. Demetriades D, Theodorou D, Cornwell EE, et al. Evaluation of penetrating injuries of the neck: Prospective study of 223 patients. World J Surg 1997;21:41-48. 
2. Feliciano D. Mattox K, Moore E. Trauma (6th ed). Editorial McGraw-Hill 2008; 469-70.

3. Demetriades D, Salim A, Brown C, Martin M, Rhee P. Neck injuries. Curr Probl Surg 2007;44:13-87.

4. Demetriades D, Theodorou D, Cornwell E, et al. Transcervical gunshot injuries: Mandatory operation is not necessary. J Trauma 1995;40:758-60.

5. Van Waes OJ, Cheriex KC, Navsaria PH, van Riet PA, Nicol AJ, Vermeulen J. Management of penetrating neck injuries. Br J Surg 2012 Jan;99 (Suppl) 1:149-54.

6. Demetriades D, Velmahos GC, Asensio JA. Penetrating injuries of the neck. In: Shoemaker W (Ed). Textbook of Critical Care (4th ed). WB Saunders, Philadelphia 2000; Chapter 29, 330-37.

7. Simmons JD, Ahmed N, Donnellan KA, Schmieg RE Jr, Porter JM, Mitchell ME. Management of traumatic vascular injuries to the neck: A 7-year experience at a level I trauma center. Am Surg 2012;78(3):335-38.

8. Inaba K, Munera F, McKenney M, Rivas L, Marecos E, de Moya $\mathrm{M}$, et al. The nonoperative management of penetrating internal jugular vein injury. J Vasc Surg 2006;43(1):77-80.

\section{ACERCA DE LOS AUTORES}

\section{Martha Quiodettis (Autor Para Correspondencia)}

Cirugía de Trauma y Cuidados Críticos Quirúrgicos, Jefa Encargada Servicio de Trauma, Hospital Santo Tomás, Panamá, e-mail: traumahst@gmail.com

\section{Otilda M Valderrama V}

Cirujana General, Servicio de Trauma, Hospital Santo Tomás, Panamá

\section{Aldo A Ávila}

Cirujano General, Servicio de Trauma, Hospital Santo Tomás, Panamá Ayusel Valle

Residente de Anestesiología, Hospital Santo Tomás, Panamá

\section{Loyda Rodríguez}

Residente de Cirugía General, Hospital Santo Tomás, Panamá 